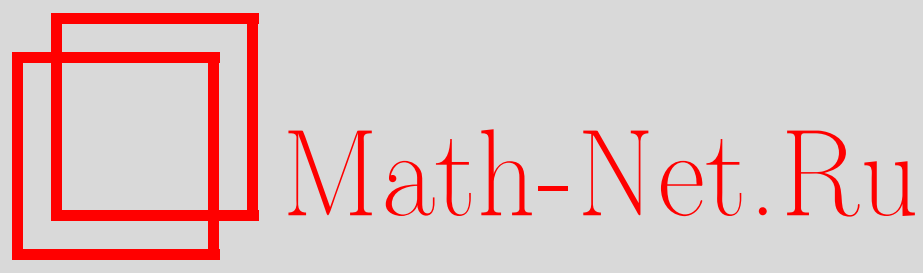

Е. Е. Тареева, Т. И. Щелкачёва, Н. М. Щелкачёв, Некоторые особенности поведения неизинговых спиновых стекол, ТМФ, 2015, том 182, номер 3, 500-512

DOI: https://doi.org/10.4213/tmf8793

Использование Общероссийского математического портала Math-Net.Ru подразумевает, что вы прочитали и согласны с пользовательским соглашением http://www . mathnet.ru/rus/agreement

Параметры загрузки:

IP : 3.89 .197 .203

26 апреля 2023 г., 13:10:35

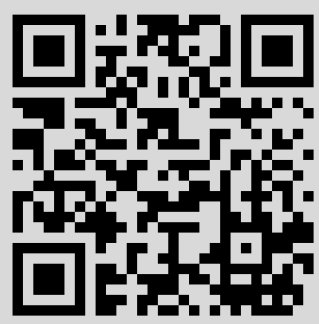




\title{
НЕКОТОРЫЕ ОСОБЕННОСТИ ПОВЕДЕНИЯ НЕИЗИНГОВЫХ СПИНОВЫХ СТЕКОЛ
}

\begin{abstract}
Работа носит обзорный характер. Дано общее представление об основных направлениях современной теории спинового стекла. Основное содержание работы основано на недавних работах авторов, которые посвящены исследованию нарушения репличной симметрии в неизинговых спиновых стеклах. Изучение целого ряда обобщенных моделей показало определенное единообразие поведения этих обобщенных спиновых стекол. Существенно, что для них наблюдается серьезное отличие от поведения известных систем со случайными связями изинговых спинов - модели Шеррингтона-Киркпатрика и соответствующей ей $p$-спиновой модели. Найдена точка ответвления решения с первым нарушением симметрии реплик, исследован вид и устойчивость решения вблизи точки ветвления, показано, в каких случаях переход в состояние стекла происходит непрерывно и в каких - со скачком параметров порядка.
\end{abstract}

Ключевые слова: спиновые стекла, квадрупольные стекла, метод реплик, модели Поттса, ветвление решений.

DOI: $10.4213 / \operatorname{tmf} 8793$

\section{1. ВВЕДЕНИЕ}

Как хорошо известно, в основе современной теории спинового стекла лежит модель Шеррингтона-Киркпатрика (ШК) [1], т. е. модель Изинга со случайными связями, со взаимодействием всех спинов со всеми, так что приближение среднего поля для нее является точным. Устойчивое решение для модели ШК было получено Паризи [2], [3] с помощью предложенного им анзаца для схемы нарушения репличной симметрии.

Естественным обобщением модели ШК явилась модель среднеполевого стекла со взаимодействием $p$ спинов, которая была предложена вскоре после появления модели ШК с парным взаимодействием спинов. В классической работе Гарднер [4] эта модель была подробно исследована. При высоких температурах устойчиво решение, описывающее парафазу так же, как и для модели ШК. Это решение совпадает

${ }^{*}$ Институт физики высоких давлений им. Л. Ф. Верещагина РАН, Троицк, Московская обл., Россия. E-mail: eetare@gmail.com

${ }^{\dagger}$ Московский физико-технический институт, Долгопрудный, Московская обл., Россия 
с нулевым реплико-симметричным (RS) решением, т. е. решением, одинаковым для всех реплик системы. При понижении температуры происходит переход в состояние стекла.

В случае $p$-спиновой модели оказалось, что имеется область температур, в которой устойчиво решение с первым этапом нарушения симметрии реплик (1RSB-решение). При этом переход от тривиального RS-решения к устойчивому $1 \mathrm{RSB}-$ решению происходит со скачком параметра упорядочения. При дальнейшем понижении температуры может иметь место переход от 1RSB-состояния к полному нарушению симметрии реплик (FRSB-состояние). Эти свойства модели резко отличают ее от модели ШК, в которой переход к FRSB-решению происходит плавно и в самой точке возникновения решения, соответствующего состоянию стекла [3]. Интересно, что исследование $p$-спиновой модели при больших $p$ показало [5], что в пределе $p \rightarrow \infty$ модель становится эквивалентной модели, рассмотренной в [6], которая решается без помощи метода реплик. Полученные двумя способами решения совпадают, что может служить косвенным оправданием не вполне обоснованного подхода Паризи.

Наличие устойчивого 1RSB-решения в некоторой области температур и скачкообразный переход к нему - важная черта $p$-спиновой модели. Дело в том, что разложение свободной энергии вблизи точки перехода в этом случае содержит члены, которые отсутствуют в модели ШК. Такие же члены присутствуют в разложении свободной энергии для двухчастичных гамильтонианов при замене изинговых спинов операторами без отражательной симметрии - это члены, содержащие нечетное число одинаковых репличных индексов. Свойства этого разложения описаны подробно в работах [7], [8].

Обычно, говоря о p-спиновой модели, имеют в виду следующий гамильтониан:

$$
H=-\sum_{i_{1} \leqslant i_{2} \leqslant \cdots \leqslant i_{p}} J_{i_{1} i_{2} \ldots i_{p}} \widehat{U}_{i_{1}} \widehat{U}_{i_{2}} \ldots \widehat{U}_{i_{p}},
$$

где $\widehat{U}_{i}$ - изинговские спины $(1,-1)$, расположенные в узлах произвольной кристаллической решетки, $N$ - число частиц. Все они связаны друг с другом элементами случайной матрицы $J_{i_{1} i_{2} \ldots i_{p}}$, распределенными по Гауссу с вероятностью

$$
P\left(J_{i_{1} i_{2} \ldots i_{p}}\right)=\frac{\sqrt{N^{p-1}}}{\sqrt{p ! \pi} J} \exp \left[-\frac{\left(J_{i_{1} i_{2} \ldots i_{p}}\right)^{2} N^{p-1}}{p ! J^{2}}\right] .
$$

Если $p=2$ и $\widehat{U}$ - спин Изинга, модель (1) переходит в обычную модель ШК [1].

Резкое различие в результатах для конфигурационной энтропии [9], полученных в случае $p$-спиновой модели и в случае модели ШК, связано именно с наличием устойчивого 1RSB-решения, которое отсутствует в модели ШК. В последней модели имеет место переход к наиболее выгодному FRSB-решению уже в точке нарушения устойчивости RS-решения.

Для исследования динамических свойств системы используют сферический вариант модели, содержащий одноузельные члены. Сферический вариант широко применяется, в частности, в связи с тем, что статические и динамические свойства такой модели можно подробно исследовать аналитически. В последние годы большое число работ было посвящено исследованию сферического варианта $p$-спиновой и $(p+s)$-спиновой моделей (сумма двух гамильтонианов с $p$ и $s$ взаимодействиями) 
и их физическим приложениям. Эти модели описывают многочисленные фазовые переходы между стекольными состояниями, начиная с 1RSB-состояния, и разнообразными последующими нарушениями симметрии реплик, в частности с полным нарушением репличной симметрии, значительно отличающимся от классического решения Паризи для модели ШК. Имеют место различные сценарии в зависимости от величин $p$ и $s$. Например, указанные модели являются прототипами систем, описывающих переходы жидкость-стекло и стекло-стекло [9]-[13]. Аналогичная модель была успешно применена при описании оптических лазерных систем при наличии беспорядка [14]. Заметим, что если в случае спинов Изинга сферический вариант гамильтониана (1) формулируется естественным и однозначным образом (поскольку $\left.s^{2}=1\right)$, то для других операторов $\widehat{U}$ - это отдельная достаточно непростая задача, не всегда разрешимая [15], [16].

Известно, что динамический анализ поведения системы можно выполнить, отталкиваясь от результатов статического подхода. С динамической точки зрения наличие макроскопического числа метастабильных состояний противодействует достижению системой стабильного состояния. Динамический переход происходит тогда, когда метастабильные состояния становятся доминирующими. Он имеет место до того, как достигается статическая критическая точка перехода. При этом различие фазовых диаграмм, построенных статически или динамически, носит главным образом количественный, а не качественный характер [17]-[20]. В работе [21] было показано, что для системы с 1RSB-структурой репличный и динамический подходы в широком смысле эквивалентны. Сферические $(s+p)$-модели предложены для описания иерархии вторичных релаксационных процессов [22]. Наличие у 1RSBмоделей перехода при низких температурах от 1RSB-решения к FRSB-решению позволяет описывать неравновесные процессы в структурных стеклах [23]-[25].

Следует заметить, что на работах по спиновым 1RSB-стеклам основаны два наиболеее популярных современных направления в так называемом равновесном подходе к реальным стеклам - это среднеполевой репличный подход (см., например, обзор [26]) и теория фазового перехода первого рода в системах со случайными связями (RFOT) [27], [28].

Таким образом, модели с $p$-взаимодействующими спинами Изинга интересны, современны и могут описывать очень широкий круг актуальных физических и математических задач [29]-[31].

Естественно, можно исследовать модели с $p$-частичным взаимодействием, рассматривая более общие операторы, нежели простейшие изинговские спины. В разделах 2-4 мы изложим наши результаты, полученные для таких обобщенных моделей. Следует заметить, что подобных работ очень немного. Отметим лишь одну из них [32]: в этой работе для случая $p \rightarrow \infty$ была рассмотрена модель, в которой $\widehat{U}=\widehat{Q}$ - операторы квадрупольного момента при $J=1$ (ср. с работой [33]). Существенно, что при замене изинговых спинов операторами без отражательной симметрии в разложении свободной энергии систем даже в случае двухчастичного взаимодействия вблизи точки ветвления присутствуют члены, которых нет в модели ШК; эти члены содержат нечетное число одинаковых репличных индексов [7], [8], [34]-[36]. При этом общая картина нарушения репличной симметрии при понижении температуры может сильно меняться (см. схему на рис. 1). 


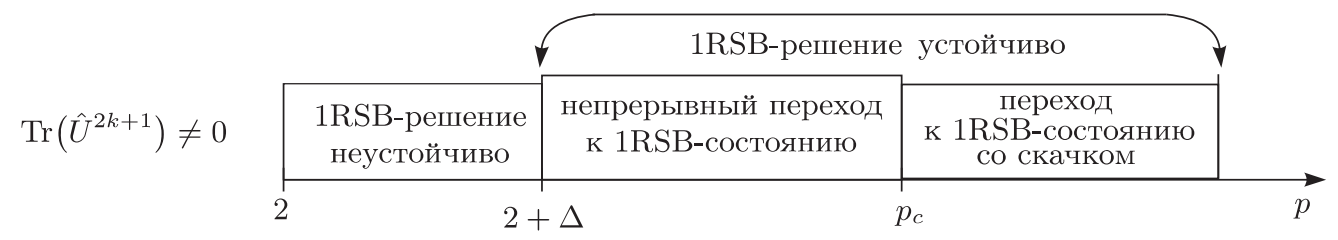

a

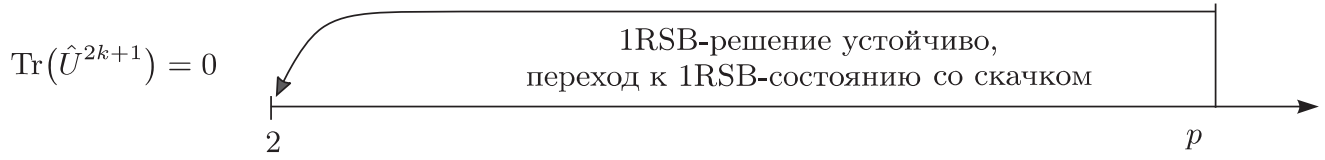

Рис. 1. Схема, показывающая качественное поведение 1RSB-решения обобщенной $p$-операторной модели в случаях, когда операторы не обладают отражательной симметрией (а) и операторы имеют отражательную симметрию, например в случае $p$-спиновой модели стекла со случайно взаимодействующими $p$ спинами Изинга (б). Здесь $p>2, k$ - целое число, $\Delta$ и $p_{\mathrm{c}}-$ величины, которые зависят от конкретного вида операторов $\widehat{U}[36]$.

Еще одним примером 1RSB-модели является модель Поттса с числом состояний, большим двух, поскольку есть область температур, при которых 1RSB-решение для этой модели устойчиво. Это свойство модели, в частности, определяет интерес к ней на протяжении последних десятилетий (см., например, работы [37]-[39]). В разделе 5 мы представим наши результаты по моделям Поттса. Существенно, что в данном случае разложение свободной энергии снова имеет тот же вид, что и для модели с $p$ взаимодействиями и произвольными $\widehat{U}$. Это позволяет, в частности, получить аналитически ряд точных результатов [40].

\section{2. ОБОЩЕННЫЕ $p$-ОПЕРАТОРНЫЕ МОДЕЛИ}

Далее мы представим некоторые результаты, полученные нами в последнее время при рассмотрении 1RSB-решений обобщенной модели стекла с $p$ взаимодействиями и моделей Поттса. Под обобщенной $p$-спиновой моделью мы будем подразумевать модель (1), где $\widehat{U}$ - произвольные диагональные операторы $(\operatorname{Tr} \widehat{U}=0)$. Конкретный вид операторов должен задаваться в соответствии с физикой задачи. Фазовая диаграмма такой модели зависит от ряда факторов, в том числе от свойств симметрии операторов $\widehat{U}$, и может оказаться в значительной степени отличной от той, которая имеет место в случае спинов Изинга.

Большинство результатов было получено при помощи методов теории ветвления решений нелинейных уравнений (см., например, монографию [41]). Этот подход оказался очень плодотворным при рассмотрении целого ряда задач. Он позволил найти точку ответвления нового решения от уже известного, описать вид решения вблизи точки ветвления, а также показать, будет ли переход в состояние стекла происходить непрерывно или со скачком параметров порядка, судить об устойчивости решения. 
Наше рассмотрение мы начнем с описания именно модели с $p$ взаимодействиями, так как рассмотрение моделей Поттса качественно аналогично. Говоря о моделях с $p$ взаимодействующими частицами, мы также имеем в виду возможность описывать как частный случай модели с парным взаимодействием при $p=2$ [42]-[44]. Мы обобщаем здесь модель с $p$ взаимодействующими частицами, считая, что $p$ меняется непрерывно при $p \geqslant 2$.

Вид высокотемпературного решения для параметра порядка зависит от свойств симметрии операторов $\widehat{U}$ [7], [34]. Важно, что в случае операторов, обладающих отражательной симметрией (когда $\operatorname{Tr} \widehat{U}^{2 k+1}=0$, где $k$ - целое число), высокотемпературное RS-решение $q_{\mathrm{RS}}$ в парафазе равно нулю. В случае $\operatorname{Tr} \widehat{U}^{2 k+1} \neq 0$ высокотемпературное RS-решение $q_{\mathrm{RS}}$ отлично от нуля при любой конечной температуре и плавно возрастает при понижении температуры. В обоих этих случаях при понижении температуры при некоторой температуре $T_{0}$ от RS-решения $q_{\mathrm{RS}}$ ответвляется новое решение, отвечающее первому нарушению репличной симметрии, которое, однако, может быть неустойчиво. Тогда при некоторой температуре $T_{1 \mathrm{RsB}}$ скачком возникает новое устойчивое стекольное решение (подробнее см. ниже).

Исследовать 1RSB-решение вблизи точки ветвления RS-решения наиболее наглядно и удобно, если непосредственно разложить выражение для свободной энергии в окрестности точки $T_{0}$. В соответствии со стандартной процедурой Паризи (для первого этапа нарушения репличной симметрии) мы делим все $n$ реплик на $n / m_{1}$ групп по $m_{1}$ реплик в каждой [3]. Параметр порядка стекла мы обозначаем как $q^{\alpha \beta}=r_{1}$, если реплики $\alpha$ и $\beta$ принадлежат различным группам, и как $q^{\alpha \beta}=r_{1}+v_{1}$, если реплики $\alpha$ и $\beta$ принадлежат к одной и той же группе.

Уравнения для стекольных параметров порядка $r_{1}, v_{1}$ и дополнительного параметра порядка $w_{1}$ получаются из условий экстремума свободной энергии. Наличие параметра $w_{1}$ связано с тем, что для произвольных операторов $\widehat{U}$ мы имеем $\widehat{U}^{2} \neq$ const, в отличие от случая спинов Изинга.

Отклонение $\Delta F$ свободной энергии $F_{1 \mathrm{RSB}}$ от еe RS-части в окрестности $T_{0}$ имеет вид

$$
\begin{aligned}
\frac{\Delta F}{N k T}= & \frac{t^{2}}{4} \frac{p(p-1)}{2} q_{\mathrm{RS}}^{p-2}\left[\lambda_{(\mathrm{RS}) \mathrm{repl}}\right]\left\{-\left[r-\left(m_{1}-1\right) v_{1}\right]^{2}-v_{1}{ }^{2} m_{1}\left(1-m_{1}\right)\right\}- \\
- & \frac{t^{4}}{2} L\left[r-\left(m_{1}-1\right) v_{1}\right]^{2}- \\
- & t^{6}\left\{C\left[r-\left(m_{1}-1\right) v_{1}\right]^{3}+D\left[r-\left(m_{1}-1\right) v_{1}\right] v_{1}{ }^{2} m_{1}\left(m_{1}-1\right)-\right. \\
& \left.\quad-B_{3} v_{1}{ }^{3} m_{1}{ }^{2}\left(m_{1}-1\right)+B_{4} v_{1}{ }^{3} m_{1}\left(m_{1}-1\right)\left(2 m_{1}-1\right)\right\}+\cdots,
\end{aligned}
$$

где $t=t_{0}+\Delta t, r=r_{1}-q_{\mathrm{RS}}$, выражения для $\lambda_{(\mathrm{RS}) \mathrm{repl}}, L, C, D, B_{3}, B_{4}$ суть средние, взятые на RS-решении в точке $T_{0}$ (подробнее см. в работе [34]). Так, например,

$$
\begin{aligned}
\lambda_{(\mathrm{RS}) \mathrm{repl}} & =1-t^{2} \frac{p(p-1) q_{\mathrm{RS}}^{p-2}}{2} \int d z^{\mathrm{G}}\left\{\frac{\operatorname{Tr}\left(\widehat{U}^{2} e^{\hat{\theta}_{\mathrm{RS}}}\right)}{\operatorname{Tr} e^{\hat{\theta}_{\mathrm{RS}}}}-\left[\frac{\operatorname{Tr} \widehat{U} e^{\hat{\theta}_{\mathrm{RS}}}}{\operatorname{Tr} e^{\hat{\theta}_{\mathrm{RS}}}}\right]^{2}\right\}^{2}= \\
& =1-t^{2}\left[\frac{p(p-1)}{2} q_{\mathrm{RS}}^{p-2}\right]\left\{\left\langle\widehat{U}_{1}^{2} \widehat{U}_{2}^{2}\right\rangle_{\mathrm{RS}}-2\left\langle\widehat{U}_{1}^{2} \widehat{U}_{2} \widehat{U}_{3}\right\rangle_{\mathrm{RS}}+\left\langle\widehat{U}_{1} \widehat{U}_{2} \widehat{U}_{3} \widehat{U}_{4}\right\rangle_{\mathrm{RS}}\right\},
\end{aligned}
$$

где

$$
\int d z^{\mathrm{G}}(\cdot)=\int_{-\infty}^{\infty} \frac{d z}{\sqrt{2 \pi}} e^{-z^{2} / 2}(\cdot), \quad t=\frac{J}{k T},
$$


оператор $\hat{\theta}_{\mathrm{RS}}$ получается из $\hat{\theta}_{1 \mathrm{RSB}}$ (см. формулу (8) ниже), если положить $v_{1}=0$. В (3) для краткости мы переходим к упрощенным обозначениям

$$
\begin{aligned}
L= & {\left[\frac{p(p-1)}{2} q_{\mathrm{RS}}^{p-2}\right]^{2}\left\{\left\langle\widehat{U}_{1}^{2} \widehat{U}_{2} \widehat{U}_{3}\right\rangle_{\mathrm{RS}}-\left\langle\widehat{U}_{1} \widehat{U}_{2} \widehat{U}_{3} \widehat{U}_{4}\right\rangle_{\mathrm{RS}}\right\} \geqslant 0, } \\
B_{3}= & {\left[\frac{p(p-1)}{2} q_{\mathrm{RS}}^{p-2}\right]^{3}\left\{\frac{1}{6}\left\langle\widehat{U}_{1}^{2} \widehat{U}_{2}^{2} \widehat{U}_{3}^{2}\right\rangle_{\mathrm{RS}}-\frac{1}{2}\left\langle\widehat{U}_{1}^{2} \widehat{U}_{2}^{2} \widehat{U}_{3} \widehat{U}_{4}\right\rangle_{\mathrm{RS}}-\right.} \\
& \left.-\frac{1}{6}\left\langle\widehat{U}_{1} \widehat{U}_{2} \widehat{U}_{3} \widehat{U}_{4} \widehat{U}_{5} \widehat{U}_{6}\right\rangle_{\mathrm{RS}}+\frac{1}{2}\left\langle\widehat{U}_{1}^{2} \widehat{U}_{2} \widehat{U}_{3} \widehat{U}_{4} \widehat{U}_{5}\right\rangle_{\mathrm{RS}}\right\} \geqslant 0,
\end{aligned}
$$

Неравенства в (5), (6) следуют из неравенства Коши-Шварца. В работе [34] было строго показано, что отклонение функции $w_{1}$ от ее RS-значения в (3) можно не учитывать в используемых нами разложениях.

В точке ветвления появляется малое отличное от нуля решение $v_{1} \rightarrow 0$ при $T \rightarrow T_{0}$. Условием возникновения такого нетривиального решения является нарушение в точке $T_{0}$ теоремы о неявной функции, т. е. отсутствие линейного по $v_{1}$ члена в соответствующем уравнении для нахождения экстремума функции (3). Отсюда получаем условие ветвления $\left.\lambda_{(\mathrm{RS}) \mathrm{rep}}\right|_{T_{0}}=0$. Будет ли равна нулю величина $L$ в $(3)$, существенно зависит от симметрии операторов $\widehat{U}$.

Из разложения (3) видно, что устойчивость RS-решения, во всяком случае вблизи точки ветвления $T_{0}$, определяется знаком $\lambda_{(\mathrm{RS}) \mathrm{repl}}$, так, при $\lambda_{(\mathrm{RS}) \mathrm{repl}}>0 \mathrm{RS}-$ решение устойчиво [7], [34], [36], [45].

Чтобы судить об устойчивости 1RSB-решения, необходимо рассмотреть дальнейшее нарушение симметрии реплик. Отсюда в качестве условия появления малого нетривиального $2 \mathrm{RSB}$-решения $v_{2} \rightarrow 0$ получаем условие бифуркации $\lambda_{(1 \mathrm{RSB}) \mathrm{repl}}=0$, где

$$
\begin{aligned}
\lambda_{(1 \mathrm{RSB}) \mathrm{repl}}= & 1-t^{2} \frac{p(p-1)\left(r_{1}+v_{1}\right)^{p-2}}{2} \times \\
& \times \int d z^{\mathrm{G}} \frac{\int d s^{\mathrm{G}} \operatorname{Tr}^{m_{1}} \exp \left(\hat{\theta}_{1 \mathrm{RSB}}\right)\left\{\frac{\operatorname{Tr}\left[\widehat{U}^{2} \exp \left(\hat{\theta}_{1 \mathrm{RSB}}\right)\right]}{\operatorname{Tr}\left[\exp \left(\hat{\theta}_{1 \mathrm{RSB}}\right)\right]}-\left[\frac{\left.\left.\operatorname{Tr}\left[\widehat{U} \exp \left(\hat{\theta}_{1 \mathrm{RSB}}\right)\right]\right]^{2}\right\}^{2}}{\operatorname{Tr}\left[\exp \left(\hat{\theta}_{1 \mathrm{RSB}}\right)\right]}\right.\right.}{\hat{\theta}_{(1 \mathrm{RSB}) \mathrm{repl}}=} \\
& z t \sqrt{\frac{\left.p r_{1}^{p-1} \exp \left(\hat{\theta}_{1 \mathrm{RSB}}\right)\right]^{m_{1}}}{2}} \widehat{U}+s t \sqrt{\frac{p\left[\left(r_{1}+v_{1}\right)^{p-1}-r_{1}^{p-1}\right]}{2}} \widehat{U}+ \\
& +t^{2} \frac{p\left[w_{1}^{p-1}-\left(r_{1}+v_{1}\right)^{p-1}\right]}{4} \widehat{U}^{2}
\end{aligned}
$$

Уравнение (7) зависит только от $1 \mathrm{RSB}-$ решения. Уравнение $\lambda_{(1 \mathrm{RSB}) \mathrm{repl}}=0$ всегда имеет решение $v_{1}=0$, которое определяет точку $T_{0}$ и совпадает с решением уравнения (4) $\lambda_{\text {(RS)repl }}=0$. Кроме точки $T_{0}$, еще одна точка бифуркации $T_{2}$, которая определяется условием $\lambda_{(1 \mathrm{RSB}) \mathrm{repl}}=0$, может существовать при $v_{1} \neq 0$. В этой точке может появиться новое 2RSB-решение. В точке $T_{2}$ может в принципе происходить и переход к решению с полным нарушением репличной симметрии. 
В точке $T_{0}$ производная $d \lambda_{(1 \mathrm{RSB}) \mathrm{repl}} / d t=0$. Таким образом, вблизи точки ветвления 1RSB-решение может быть как устойчивым, так и неустойчивым, в зависимости от конкретного вида операторов $\widehat{U}$ и значения параметра $p$. Решение может быть устойчивым по отношению к возбуждениям, имеющим 2RSB-симметрию, при $\lambda_{(1 \mathrm{RSB}) \mathrm{repl}}>0$ [34]. Выражение для $\Delta F_{2}=F_{\text {2RSв }}-F_{1 \mathrm{RSB}}$ при произвольной температуре можно представить в виде суммы квадратов малых отклонений соответствующих параметров порядка от параметров для 1RSB-случая. При этом член разложения, содержащий $v_{2}^{2}$, пропорционален $-\lambda_{(1 \mathrm{RSB}) \mathrm{repl}}$.

\section{3. ОБЩИЕ РЕЗУЛЬТАТЫ}

Итак, уравнения для 1RSB-параметров порядка в окрестности $T_{0}$ получаются из условий экстремума для $\Delta F$ (см. формулу (3)). В зависимости от свойств симметрии операторов $\widehat{U}$ мы имеем два разных сценария. Поскольку $q_{\mathrm{RS}}=0$ в случае $\operatorname{Tr} \widehat{U}^{2 k+1}=0$, в точке $T_{0}$ получаем из (6) $L=0$, а также $C=-D=3 B_{3}, B_{4}=0$ [34]. Из условия ветвления $\lambda_{(\mathrm{RS}) \mathrm{repl}}=0$ и выражения (4) следует, что, поскольку $q_{\mathrm{RS}}=0$, только при $p=2$ возникновение $1 \mathrm{RSB}$-состояния происходит плавно при конечном значении $t$, и мы получаем из $(3)$ вблизи $T_{0}$ при $p=2$ следующие уравнения:

$$
\begin{gathered}
m_{1}=0, \\
r_{1}+v_{1}=\frac{1+t_{0}^{4}\left(w_{\mathrm{RS}}+\left(t_{0} / 2\right) \dot{w}_{\mathrm{RS}}\right)\left[\left\langle\widehat{U}_{1}^{2} \widehat{U}_{2}^{4}\right\rangle_{\mathrm{RS}}-\left\langle\widehat{U}_{1}^{2} \widehat{U}_{2}^{2} \widehat{U}_{3}^{2}\right\rangle_{\mathrm{RS}}\right]}{\left\langle\widehat{U}_{1}^{2} \widehat{U}_{2}^{2} \widehat{U}_{3}^{2}\right\rangle_{\mathrm{RS}} t_{0}^{5}} \Delta t,
\end{gathered}
$$

где $\dot{w}_{\mathrm{RS}}=d w_{\mathrm{RS}} / d t, \Delta t=t-t_{0}$.

При других значениях параметра $p$ 1RSB-решение возникает скачком в точке $T_{1 \mathrm{RSB}}$, в которой $m\left(T_{1 \mathrm{RSB}}\right)=1$. Таким образом, имеет место такая же фазовая диаграмма, как и в случае обычной модели со спинами Изинга.

В противоположность этому случаю при $\operatorname{Tr} \widehat{U}^{2 k+1} \neq 0$ решение с нарушенной симметрией реплик может возникать непрерывным образом не только в случае парных взаимодействий, $p=2$, но и при любых конечных $p>2$. Причиной этого является отсутствие тривиального RS-решения. При этом условие $\lambda_{(\mathrm{RS}) \mathrm{repl}}=0$ возможного появления 1RSB-решения удовлетворяется при температуре $T_{0} \neq 0$. Это решение появляется плавно, если $m_{1}\left(T_{0}\right) \leqslant 1$. В противном случае имеющее физический смысл $1 \mathrm{RSB}$-решение возникает скачком при температуре $T_{1 \mathrm{RSB}}$, когда достигается значение $m_{1}=1$. Алгебраическое выражение для $m_{1}\left(T_{0}\right)$ зависит только от RSрешения в точке $T_{0}$.

Итак, в случае, когда $\operatorname{Tr} \widehat{U}^{2 k+1} \neq 0$ при $k \neq 0$, параметр порядка стекла $q_{\mathrm{RS}} \neq 0$ для любого конечного значения температуры $T$. Таким образом, получаем из (6), что $\left.L\right|_{t=t_{0}} \neq 0$. Далее, из условий экстремума для свободной энергии по параметрам порядка с учетом того, что в точке ветвления $\lambda_{(\mathrm{RS}) \text { repl }}=0$, мы получаем из (3) дополнительное условие ветвления:

$$
r-\left(m_{1}-1\right) v_{1}=0+o(\Delta t)^{2}
$$

1RSB-решение всегда плавно ответвляется от RS-решения при некоторой конечной температуре $T_{0}$. 
Из условий экстремума получаем

$$
\begin{aligned}
& 2 Z \Delta T=3 t_{0}^{6} m_{1}\left(m_{1}-1\right) B_{3} v_{1}, \\
& Z=\frac{t^{2}}{4} \frac{p(p-1)}{2} \frac{d\left[q_{\mathrm{RS}}^{p-2} \lambda_{(\mathrm{RS}) \mathrm{rep} 1}\right]}{d T} .
\end{aligned}
$$

При этом

$$
m_{1}=\frac{B_{4}}{B_{3}}
$$

в точке ветвления $T_{0}$, где $1 \mathrm{RSB}$-решение возникает. При этом значения $B_{3}$ и $Z$ берутся в точке $T=T_{0}$. Случаи $m_{1}=0$ и $m_{1}=1$ следует рассматривать отдельно. В результате в окрестности точки $T_{0}$ мы имеем

$$
r_{1}=q_{\mathrm{RS}}+\left(m_{1}-1\right) v_{1}, \quad v_{1} \sim T-T_{0} .
$$

Как можно видеть из уравнения (12), величина параметра $m_{1}$ в точке $T_{0}$ определяет поведение параметра порядка стекла $v_{1}$. Как в случае $0<m_{1}<1$, так и при $m_{1}>1$ имеет место линейная зависимость от $\Delta T=T-T_{0}$, но знак коэффициента пропорциональности зависит от $m_{1}$. Действительно, функция $\lambda_{(\mathrm{RS}) \mathrm{rep}}$ возрастает с ростом температуры. Она равна нулю при $T=T_{0}$ и изменяет знак в этой точке, так как знак $\lambda_{(\mathrm{RS}) \text { repl }}$ определяет устойчивость RS-решения. Параметр порядка $q_{\mathrm{RS}}$ является положительной и медленно меняющейся функцией от температуры в окрестности $T=T_{0}$. Отсюда мы получаем, что $Z>0$ вблизи точки $T_{0}$ (см. формулу (13)).

Когда $0<m_{1}<1$, решение идет влево от точки $T_{0}$, таким образом, $1 \mathrm{RSB}$-решение возникает плавно. Решение может быть устойчиво в области $\lambda_{(1 \mathrm{RsB}) \mathrm{repl}}>0$. Во втором случае, когда $m_{1}>1$, положительное решение имеет место для $T \geqslant T_{0}$. Это решение является нефизическим вблизи $T=T_{0}$, так как значения $m_{1}>1$ не имеют физического смысла. Таким образом, 1RSB-решение может возникнуть только скачком, когда функция $m_{1}(T)$ достигает значения $m_{1}(T)=1$ при некоторой температуре $T_{1 \mathrm{RSB}}$.

Если мы получим $m_{1}=1$ при $T=T_{0}$ для какого-либо значения $p_{\text {c }}$, то таким образом мы найдем точку, в которой происходит переход от плавного к скачкообразному 1RSB-решению. В этом случае необходимо рассматривать члены четвертого порядка в разложении свободной энергии (3). Можно показать, что в этом случае $v_{1}^{2} \sim T_{0}-T$

\section{4. ПРИМЕР КВАДРУПОЛЬНОГО СТЕКЛА}

В качестве простейшего примера системы с $p$ взаимодействиями и операторами без отражательной симметрии рассмотрим квадрупольное стекло при $J=1$. В этом случае $\widehat{U}=\widehat{Q}=3 J_{z}^{2}-2$ есть аксиальный квадрупольный момент молекулы. Квадрупольный момент принимает значения $-2,1,1$. Мы провели расчеты для модели с различными значениями $p$, которые могут меняться непрерывным образом.

При $p<2+\Delta p$, где $\Delta p=0.5$ является конечным числом, решение качественно ведет себя так же, как и в случае парного взаимодействия. При $p<2.5$ 1RSB-решение становится нестабильным, так как $\lambda_{(1 \mathrm{RsB}) \text { repl }} \leqslant 0$ для $T<T_{0}$. Такое поведение естественно из соображений непрерывности. 


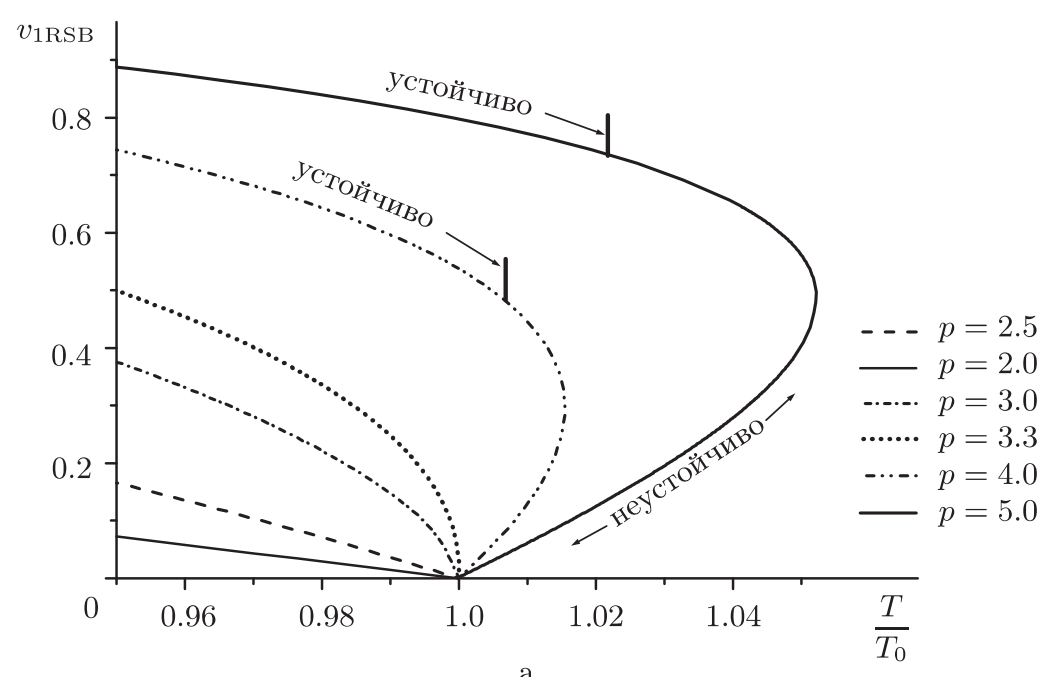

a

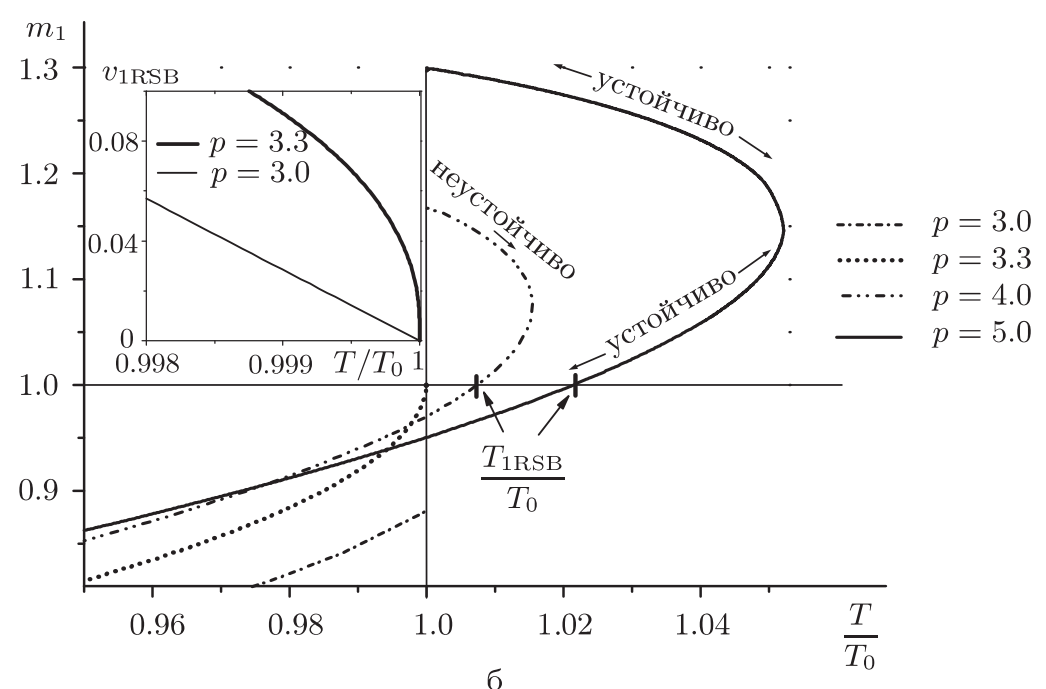

Рис. 2. Поведение параметров порядка как функций температуры для $p$-квадрупольной модели. При $p=3.3$ происходит переход от непрерывного к скачкообразному сценарию перехода в состояние стекла; $m_{1}=1$ в точке ветвления $T_{0}, v_{1} \backsim \sqrt{T_{0}-T}$ вблизи $T_{0}$ [36].

При $p=3$ устойчивое $1 \mathrm{RSB}$-решение появляется в точке бифуркации $T_{0}=T_{1 \mathrm{RSB}}$ и продолжается непрерывным образом в сторону температур, меньших чем $T_{0}$ (см. рис. 2). В точке бифуркации $m_{1}=0.88<1$. Из равенства (12) видно, что в окрестности точки ветвления $v_{1} \backsim-\left(T-T_{0}\right)$. При $p=4,5, \ldots$ RSB-решение появляется непрерывным образом в точке ветвления $T_{0}$, которая определяется условием $\lambda_{(\mathrm{RS}) \mathrm{repl}}=0$. Но это решение нефизично в окрестности $T=T_{0}$. Дело в том, что $m_{1}>1$ (для $p=4$ мы получили $m_{1}=1.17$, а для $p=5$ мы получили $m_{1}=1.3$ ) 


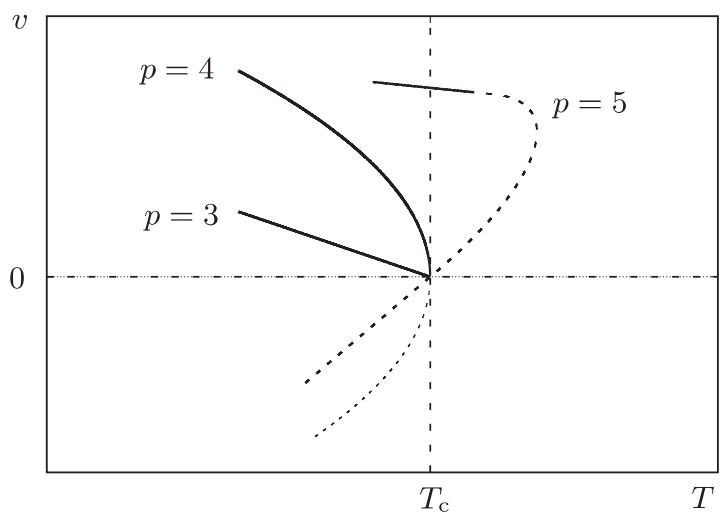

Рис. 3. Схематическое изображение поведения стекольного параметра порядка $v_{1}$ вблизи точки бифуркации для моделей Поттса при $p=3,4,5$. Сплошные линии соответствуют решению, для которого $m_{1} \leqslant 1$, а штриховые линии соответствуют нефизичной части решения с $m_{1}>1$ [40].

в точке ветвления, что не имеет физического смысла. Из формулы (12) выводим, что $v_{1} \backsim T-T_{0}$ вблизи $T=T_{0}$. Переход от RS- к $1 \mathrm{RSB}-$ решению происходит скачком в точке $T_{1 \mathrm{RSB}}>T_{0}$, которая определяется условием $m_{1}=1$. В этой точке свободная энергия $F_{\mathrm{RS}}=F_{1 \mathrm{RSB}}$. Репликосимметричное решение стабильно при температурах выше $T_{1 \mathrm{RSB}}$. При этом $m_{1}<1$ при $T<T_{1 \mathrm{RSB}}$, и соответствующее $1 \mathrm{RSB}$-решение имеет физический смысл, поскольку отвечает более выгодному большему значению свободной энергии, чем RS-решение (см. рис. 3).

При $p=3.3$ имеет место кроссовер от непрерывного к скачкообразному сценарию. В этом случае $m_{1}=1$ в точке ветвления. Правая часть уравнения (12) равна нулю. Необходимо рассматривать следующие члены в разложении (3) в окрестности значения $T_{0}$. Получаем, что вблизи точки перехода $v_{1} \backsim \sqrt{T_{0}-T}$.

\section{5. МОДЕЛЬ ПОТТСА}

Модель Поттса определяется гамильтонианом

$$
\widehat{H}=-\frac{p}{2} \sum_{i \neq j} J_{i j} \delta_{\sigma_{i}, \sigma_{j}},
$$

где $J_{i j}$ - распределенные по Гауссу случайные переменные, $p$ - число состояний и $\sigma_{i}, \sigma_{j}$ принимают значения $0,1, \ldots, p-1$. При $p=2$ модель Поттса сводится к модели ШК.

Подобное предыдущему описание и поведение параметров порядка имеют место и в случае моделей стекла Поттса для $p>2$. Случай 3,4 и 5 состояний рассмотрен в работе [40]. Дело в том, что разложение (3) для $\Delta F$ качественно одно и то же для моделей (1) и (16).

При значениях $p=3,4$ переход парафаза-устойчивое стекольное $1 \mathrm{RSB}$-решение происходит непрерывным образом в само́й точке ответвления нового решения с первым нарушением симметрии реплик от тривиального решения. При $p>4$ переход к новому решению происходит скачкообразно. Существенным в данном случае 
оказалось то, что свободную энергию и 1RSB-решение для модели Поттса удобно описывать аналогично соответствующему рассмотрению для модели с $p$ взаимодействиями и произвольными операторами $\widehat{U}$ (см. работу [40]). Это позволяет, в частности, получить аналитически ряд точных результатов в отличие от предыдущих численных [38].

Для модели Поттса с $p=3$ удобно для вычислений и наглядно использовать следующее представление [46]-[48]:

$$
H=-\frac{1}{2} \sum_{i \neq j} J_{i j}\left[\widehat{Q}_{i} \widehat{Q}_{j}+\widehat{V}_{i} \widehat{V}_{j}\right],
$$

где $\widehat{Q}=3 J_{z}^{2}-2$ есть аксиальный квадрупольный момент при $J=1$ и $J_{z}=0, \pm 1$, принимающий значения $-2,1,1$; оператор $\widehat{V}=\sqrt{3}\left(J_{x}^{2}-J_{y}^{2}\right)$ - неаксиальная компонента квадрупольного момента, $\widehat{V}=0, \pm \sqrt{3}$. Оператор $\widehat{Q}$ не обладает отражательной симметрией, а $\widehat{V}$ обладает. Эквивалентность формул (16) и (17) следует из соотношения

$$
\left[Q_{k} Q_{l}+V_{k} V_{l}\right]+2=6 \delta_{k, l}
$$

В случае стекла Поттса отражательная симметрия отсутствует. Мы имеем отличные от нуля кубические члены:

$$
\begin{aligned}
B_{4} & =\frac{1}{12}\left[\operatorname{Tr}^{2} \frac{Q^{3}}{3}+3 \operatorname{Tr}^{2} \frac{Q V^{2}}{3}\right]=\frac{4}{3}, \\
B_{3} & =\frac{1}{6}\left[\operatorname{Tr}^{3} \frac{Q^{2}}{3}+\operatorname{Tr}^{2} \frac{V^{2}}{3}\right]=\frac{8}{3}, \\
D & =-4 .
\end{aligned}
$$

Отсюда $B_{4} / B_{3}=m_{1}=1 / 2$ в точке ветвления. Переход происходит без скачка параметра порядка. Как было показано в работе [49], 1RSB-решение устойчиво в окрестности точки перехода; при понижении температуры оно становится неустойчивым и происходит следующий этап нарушения репличной симметрии.

Важно отметить, что симметрия моделей Поттса приводит к отсутствию некоторых членов в выписанном в предыдущем разделе разложении свободной энергии. Из (6) получаем $\left.L\right|_{T=T_{0}}=0$, так как $r_{1}=r=0$ [38] вследствие симметрии модели Поттса. Таким образом, ветвление происходит от нулевого RS-решения. Это резко отличает модели Поттса от обобщенных $p$-спиновых моделей в случае операторов $\widehat{U}$, не имеющих отражательной симметрии. Поскольку $\left.L\right|_{T=T_{0}}=0$, условие $r-\left(m_{1}-1\right) v_{1}=0$ не удовлетворяется, но уравнения типа (12) и (14) имеют место.

В работе [40] с помощью использования соответствующих представлений были описаны модели Поттса с $p=3,4,5$. При $p=3$ и $p=5$ зависимость $v_{1}$ от температуры имеет линейный характер в окрестности точки ветвления, но параметры порядка в этих двух случаях имеют разный знак. Значение $p=4$ является граничным, при котором переход к $1 \mathrm{RSB}$-решению непрерывен. При этом в точке ветвления $m_{1}=1$ и $v_{1} \backsim \sqrt{T_{0}-T}$ в окрестности $T_{0}$.

Таким образом, последовательное применение методов теории ветвлений к решениям нелинейных уравнений, выражающих условие экстремума свободной энергии, позволило получить ряд новых результатов. Были изучены обобщенные модели спинового стекла со случайным взаимодействием $p$ произвольных операторов 
и некоторые модели Поттса. Показано, что во многих случаях картина нарушения репличной симметрии (устойчивость решений, непрерывность и др.) сильно отличается от обычной ситуации, которая имеет место в системах изинговых спинов со случайными связями.

Благодарности. Е. Е. Тареева (разделы 1, 5) благодарна за поддержку РНФ (грант № 14-22-00093). Т. И. Щелкачёва (раздел 2) выражает благодарность РФФИ (грант № 14-02-00451). Н. М. Щелкачёв (разделы 3, 4) был поддержан РНФ (грант № 14-12-01185).

\section{Список литературы}

[1] D. Sherrington, S. Kirkpatrick, Phys. Rev. Lett., 35:26 (1975), 1792-1796; S. Kirkpatrick, D. Sherrington, Phys. Rev. B, 17:11 (1978), 4384-4403.

[2] G. Parisi, J. Phys. A: Math. Gen., 13:4 (1980), L115-L121; 13:3 (1980), 1101-1112; 13:5 (1980), 1887-1895.

[3] M. Mézard, G. Parisi, M. Virasoro, Spin Glass Theory and Beyond, World Sci., Singapore, 1987.

[4] E. Gardner, Nucl. Phys. B, 257:6 (1985), 747-765.

[5] D. Gross, M. Mézard, Nucl. Phys. B, 240:4 (1984), 431-452.

[6] B. Derrida, Phys. Rev. Lett., 45:2 (1980), 79-82; Phys. Rev. B, 24:5 (1981), 2613-2626.

[7] Е. Е. Тареева, Т. И. Щелкачёва, Н. М. Щелкачёв, ТМФ, 160:2 (2009), 385-400.

[8] T. I. Schelkacheva, E. E. Tareyeva, N. M. Chtchelkatchev, Phys. Rev. B, 82:13 (2010), 134208, 5 pp., arXiv: 1005.4923.

[9] A. Crisanti, L. Leuzzi, T. Rizzo, Phys. Rev. B, 71:9 (2005), 094202, 11 pp., arXiv: cond-mat/0406649.

[10] A. Crisanti, L. Leuzzi, G. Parisi, T. Rizzo, Phys. Rev. Lett., 92:12 (2004), 127203, 4 pp., arXiv: cond-mat/0307543.

[11] A. Crisanti, L. Leuzzi, Phys. Rev. B, 76:18 (2007), 184417, 8 pp., arXiv: 0705.3175.

[12] A. Crisanti, L. Leuzzi, Phys. Rev. B, 73:1 (2006), 014412, 20 pp.

[13] A. Crisanti, L. Leuzzi, Phys. Rev. Lett., 93:21 (2004), 217203, 4 pp., arXiv: cond-mat/0407129.

[14] F. Antenucci, C. Conti, A. Crisanti, L. Leuzzi, General phase-diagram of multimodal ordered and disordered lasers in closed and open cavities, arXiv: 1406.7826.

[15] N. V. Gribova, V. N. Ryzhov, E. E. Tareyeva, Phys. Lett. A, 353:2-3 (2006), 226-229.

[16] Е. Е. Тареева, ТМФ, 168:3 (2011), 584-592.

[17] T. Kirkpatrick, D. Thirumalai, Phys. Rev. Lett., 58:20 (1987), 2091-2094.

[18] A. Crisanti, H. Horner, H.-J. Sommers, Z. Phys. B, 92:2 (1993), 257-271.

[19] L. Bouchaud, J. P. Cugliandolo, J. Kurchan, M. Mézard, Physica A: Statist. Mech. Appl., 226:3 (1996), 243-273, arXiv: cond-mat/9511042.

[20] F. Caltagirone, U. Ferrari, L. Leuzzi, G. Parisi, F. Ricci-Tersenghi, T. Rizzo, Phys. Rev. Lett., 108:8 (2012), 085702, 5 pp., arXiv: 1111.6420.

[21] A. Crisanti, Nucl. Phys. B, 796:3 (2008), 425-456, arXiv: 0710.0601.

[22] A. Crisanti, L. Leuzzi, A simple spin model for three steps relaxation and secondary processes in glass formers, arXiv: 1405.6832 .

[23] P. Charbonneau, J. Kurchan, G. Parisi, P. Urbani, F. Zamponi, Nature Commun., 5 (2014), 3725, 6 pp., arXiv: 1404.6809.

[24] T. Rizzo, Phys. Rev. E, 88:3 (2013), 032135, 12 pp., arXiv: 1305.2070.

[25] G. Parisi, F. Ricci-Tersenghi, T. Rizzo, J. Stat. Mech., 4 (2014), P04013, 21 pp.

[26] G. Parisi, F. Zamponi, Rev. Modern Phys., 82:1 (2010), 789-845. 
[27] P. G. Wolynes, V. Lubchenko, Structural Glasses and Supercooled Liquids: Theory, Experiment, and Applications, Wiley, New York, 2012.

[28] L. Berthier, G. Biroli, Rev. Modern Phys., 83:2 (2011), 587-645, arXiv: 1011.2578.

[29] D. Panchenko, Ann. Probab., 42:3 (2014), 946-958.

[30] M. Talagrand, Mean Field Models for Spin Glasses, v. 1: Basic Examples, Ergebnisse der Mathematik und ihrer Grenzgebiete. 3. Folge / A Series of Modern Surveys in Mathematics, 54, Springer, Berlin, 2011.

[31] F. Caltagirone, U. Ferrari, L. Leuzzi, G. Parisi, T. Rizzo, Phys. Rev. B, 83:10 (2011), 104202, 8 pp., arXiv: 1010.5000.

[32] K. Walasek, J. Phys. A: Math. Gen., 28:18 (1995), L497-L501.

[33] T. I. Schelkacheva, E. E. Tareyeva, N. M. Chtchelkatchev, Phys. Rev. E, 79:2 (2009), 021105, 7 pp., arXiv: 0809.0877.

[34] T. I. Schelkacheva, N. M. Chtchelkatchev, J. Phys. A: Math. Theor., 44:44 (2011), 445004, 14 pp., arXiv: 1103.4308.

[35] E. E. Tareyeva, T. I. Schelkacheva, N. M. Chtchelkatchev, J. Phys. A: Math. Theor., 47:7 (2014), 075002, 12 pp., arXiv: 1309.4292.

[36] T. I. Schelkacheva, E. E. Tareyeva, N. M. Chtchelkatchev, Phys. Rev. E, 89:4 (2014), 042149, 5 pp., arXiv: 1309.6987.

[37] D. J. Gross, I. Kanter, H. Sompolinsky, Phys. Rev. Lett., 55:3 (1985), 304-307.

[38] E. De Santis, G. Parisi, F. Ritort, J. Phys. A: Math. Gen., 28:11 (1995), 3025-3041.

[39] V. Janiš, A. Klíč, Phys. Rev. B, 84:6 (2011), 064446, 10 pp.

[40] N. V. Gribova, T. I. Schelkacheva, E. E. Tareyeva, J. Phys. A: Math. Theor., 43:49 (2010), 495006, 9 pp.

[41] М. М. Вайнберг, В.А. Треногин, Теория ветвления решений нелинейных уравнений, Наука, М., 1969.

[42] М. Г. Васин, Е.Е. Тареева, Т. И. Щелкачёва, Н. М. Щелкачёв, ТМФ, 174:2 (2013), $228-242$.

[43] Е. Е. Тареева, Т. И. Щелкачёва, Н. М. Щелкачёв, ТМФ, 155:2 (2008), 356-368.

[44] В. Н. Рыжов, Е.Е. Тареева, Т. И. Щелкачёва, Н. М. Щелкачёв, ТМФ, 141:1 (2004), 131-140.

[45] J. R. L. de Almeida, D. J. Thouless, J. Phys. A, 11:5 (1978), 983-990.

[46] Е. А. Лучинская, Е. Е. Тареева, ТМФ, 87:3 (1991), 473-480.

[47] Е. А. Лучинская, Е. Е. Тареева, ТМФ, 91:1 (1992), 157-167.

[48] E. A. Lutchinskaia, E. E. Tareyeva, Europhys. Lett., 17:2 (1992), 109-112.

[49] N. V. Gribova, V. N. Ryzhov, E. E. Tareyeva, Phys. Rev. E, 68:6 (2003), 067103, 4 pp., arXiv: cond-mat/0307042. 